\title{
Violencia Psicológica en la relación de noviazgo en estudiantes universitarios que inician la carrera de Psicología. Un estudio cualitativo
}

\author{
Psychological Violence in the dating relationship in university students starting the \\ Psychology career. A qualitative study
}

\begin{abstract}
Resumen
Introducción: el alarmante incremento de la violencia en las parejas de novios produce devastadoras consecuencias psicológicas y comportamentales. Objetivo: comprender, desde una perspectiva cualitativa, las características de la violencia psicológica en la relación de noviazgo, respecto a sus significados, percepción, modalidades, y respuestas hacia la misma, en estudiantes de inicio de carrera de la Facultad de Psicología de una universidad privada de Mendoza. Método: Investigación cualitativa, estudio descriptivo, diseño fenomenológico. Muestra intencional conformada por alumnos de $1^{\circ}$ año de la carrera Licenciatura en Psicología y Licenciatura en Niñez, Adolescencia y Familia ( $\mathrm{n}=74)$, de ambos sexos, en un rango de edad de 18 a 21 años, que hubieran
\end{abstract}

María de los Ángeles Páramo*

Flavia Susana Arrigoni** Aldana Romina Ligorria Diaz***

mantenido una relación de noviazgo. Se utilizó triangulación de técnicas: grupos de discusión sobre la violencia psicológica con una guía de 17 líneas temáticas $(\mathrm{n}=11)$ y redes semánticas naturales. Para el análisis cualitativo se utilizó el software TextSTAT versión 2.9c. Resultados: del análisis de las redes semánticas naturales se desprende que la violencia psicológica es definida con las palabras "maltrato", "agresión", "manipulación", "control”, "insultos" y "gritos", causando "dolor" y "miedo". En los grupos de discusión se consideraron las dos palabras clave más significativas y frecuentes en cada categoría de análisis, entre las que se encontraron: "ser", "no", "estar", “yo", "persona", "poder”, "mucho", "violencia", "psicológica", "sentir", "celos", "culpa", "cosa", "creer", "otro" y "mujer".

Conclusión: la violencia psicológica es "más

* Dra. en Psicología Clínica y de la Salud. Universidad del Aconcagua, Mendoza, Argentina. Mail de contacto: maparamo@uda.edu.ar

** Master en Intervención Psicológica en Contextos de Riesgo. Universidad del Aconcagua, Mendoza, Argentina ; Pontificia Universidad Católica Argentina, Buenos Aires, Argentina. Mail de contacto: farrigoni@uda.edu.ar *** Lic. en Psicología. Universidad del Aconcagua, Mendoza, Argentina ; Pontificia Universidad Católica Argentina, Buenos Aires, Argentina. Mail de contacto: aldanaldiaz@, uda.edu.ar DOI: https://doi.org/10.46553/RPSI.17.33.2021.p36-60

Fecha de recepción: 8 de agosto de 2020 - Fecha de aceptación: 8 de marzo de 2021 
constante" y antecede a la física "primero se da la violencia psicológica y después se da la física". Urge instrumentar estrategias de prevención de la violencia relacional desde edades tempranas.

Palabras clave: violencia psicológica, noviazgo, estudiantes universitarios, estudio cualitativo.

\begin{abstract}
Introduction. The alarming increase in dating violence produces devastating psychological and behavioral consequences. Objective: To understand, from a qualitative perspective, the characteristics of psychological violence $(\mathrm{PV})$ in the dating relationship, regarding its meanings, perception, modalities, and response to it, in students at the beginning and end of their studies at the Faculty of Psychology of a private university in Mendoza. Method: Qualitative research, descriptive study, phenomenological design. Intentional sample made up of students from the first course in the careers Psychology and Childhood, Adolescence and Family $(n=74)$, of both sexes, age range from 18 to 21 years old, who had been in a dating relationship. The triangulation of techniques was used: discussion groups $(\mathrm{n}=11)$, semantic networks and TextSTAT software version $2.9 \mathrm{c}$. for the analysis of information. Results: in the analysis of semantic networks, it is observed that psychological violence is defined with the words "abuse", "aggression", "manipulation", "control", "insults" and "screaming", causing "pain" and "fear". In the discussion groups, the two most significant and frequent keywords were considered in each category of analysis, among which the following words were found: "to be", "not", "I", "person", "power",
\end{abstract}

"a lot", "violence", "psychological", "feel", "jealousy", "guilt", "thing", "believe", "other" and "woman". Conclusion: psychological violence is "more constant" and precedes physical violence "psychological violence occurs first and then physical violence occurs." It is urgent to implement strategies to prevent dating violence from an early age. Key words: psychological violence, dating relationship, bachelor students, qualitative study

\section{Introducción}

El noviazgo, relación entre dos personas para "acompañarse en las actividades recreativas y sociales, y en la cual se expresan sentimientos amorosos y emocionales a través de la palabra y los contactos corporales" (Rodríguez \& de Keijzer, 2002, en Vásquez García y Castro, 2008, p. 715), puede estar signado por la violencia, entendiendo por tal a "todo acto, omisión, actitud o expresión que genere, o tenga el potencial de generar daño emocional, físico o sexual a la pareja afectiva con la que se comparte una relación íntima sin convivencia ni vínculo marital" (Castro y Casique, 2010 citado en Rojas Solís, 2013, p.50).

Con el paso del tiempo, las necesidades de estudio sobre el tema de la violencia en la pareja han cambiado, al hacerse presente no sólo en personas adultas, sino cada vez más en jóvenes y fuera del vínculo matrimonial (Peña Cárdenas et al. 2013). Una investigación realizada en España muestra que en el 22\% de los casos, los problemas empiezan durante el noviazgo (Hernando Gómez et al., 2012). "Ha sido una creencia socialmente aceptada que el noviazgo es la época ideal de una pareja, sin 
embargo, la realidad se muestra diferente porque las jóvenes parejas muchas veces se enfrentan a situaciones violentas", es lo que plantean Alegría del Ángel y Rodríguez Barraza (2015, p. 58).

Por su parte Guzmán-González et al. (2014) establecen que las dinámicas de violencia existentes en las relaciones de noviazgo pueden clasificarse en tres tipos: física, entendida como acciones no accidentales que buscan agredir el cuerpo de la pareja; sexual, definida como cualquier acto sexual o tentativa de consumarlo sin que sea consentido; y psicológica, considerada como tal la desvalorización, intimidación, desprecio y la humillación tanto en público como en privado hacia una pareja.

A su vez, para Toldos Romero (2013, p. 26) violencia psicológica (VP) se refiere a "todas aquellas conductas, actos o exposición a situaciones que ocasionen o puedan ocasionar daño emocional o perturbar la existencia y desarrollo sano de la víctima".

Si bien la violencia psicológica es la forma de agresión más reconocida por la juventud (Rey-Anacona, 2013), ha sido, paradójicamente, el tipo de violencia menos investigado (Moura Lourenço et al., 2013). La violencia que ocurre dentro de la pareja de novios ha evidenciado un preocupante aumento y sus consecuencias psicológicas y comportamentales son altamente nocivas (Wolfe, Crooks \& Hughes, 2011).

Aun hoy prevalece cierto interés por la dimensión física del maltrato, aún a pesar de que Rey-Anacona (2013) indica que las agresiones de tipo psicológico se presentan antes que las de tipo físico. "Ejercer control sobre el otro/a, es una cuestión que trasciende a la mera agresión física, identificando el incremento progresivo de patrones de interacción coactiva en la pareja como variables anticipadoras de conductas de violencia física en la pareja" (Blázquez Alonso et al., 2015, párr.3).

En relación a la violencia en el noviazgo, por lo general, los jóvenes estudiantes "reconocen como violencia sólo la agresión física, el golpe o la marca en el cuerpo" (Di Franco et al., 2016, p.52), quizás debido a la impulsividad, la emocionalidad o el deficitario autocontrol característico de estas edades (Rojas Solís, 2013).

Rey-Anacona (2009) realizó un estudio exploratorio de maltrato de tipo físico, psicológico, emocional, sexual y económico en el noviazgo, haciendo evidente la presencia de todos estos tipos de violencia. El autor y su equipo consideraron las diferencias por sexos, y encontraron que, si bien los varones ejercieron más conductas de maltrato emocional, sexual, económico y negligente que las mujeres, no se encontraron diferencias significativas entre sexos con respecto al maltrato físico y psicológico (Rey-Anacona et al., 2010). Al estudiar las conductas de maltrato entre adolescentes de áreas urbanas y rurales, se observó que la frecuencia de comportamientos de maltrato fue significativamente mayor entre los/as del área urbana, tanto en general como en lo concerniente a los tres tipos de maltrato: psicológico, emocional y físico (Rey Anacona et al., 2017).

Olvera Rodríguez et al. (2012) muestran que el $92 \%$ de las estudiantes universitarias que participaron en su investigación reconoció indicios de violencia psicológica en la inmediata relación pasada o en la que actualmente mantenían. El $34 \%$ manifestó vivir episodios de violencia psicológica combinada con alguno de los 
otros tipos de violencia - física, económica y sexual-, y solo un $2 \%$ no apreció ningún indicio de violencia en su relación.

Con respecto a la edad, hay estudios que han encontrado que las mujeres más jóvenes tienen menos riesgo de sufrir maltrato en el noviazgo que las de más edad, concluyendo que este tipo de abuso comienza en edades posteriores (Sabina et al., 2016, citado en Redondo Pacheco et al., 2017). Sin embargo, Tolan et al. (2006, citado en Guzmán-González et al., 2014) indicaron en su estudio que la etapa que va de los 20 a los 24 años es la que tiene más alta tasa de riesgo de sufrir violencia.

El estudio de la violencia en las relaciones de noviazgo es importante en la comprensión global del fenómeno de la violencia en las relaciones íntimas, no solamente porque produce lesiones y sufrimiento, tanto en jóvenes como en personas adultas, sino porque su conocimiento puede ayudar en la planeación de estrategias encaminadas al abordaje y a su prevención (Peña Cárdenas et al. 2013).

Urbiola et al. (2019) clasifican los tipos de VP en las relaciones de pareja, hablan de la violencia psicológica social, de humillación y de control. Esta última alude a los celos, al control de las redes sociales, de las amistades y de las formas de vestir de la pareja, entre otras conductas ejemplificadoras; y la VP de humillación se manifiesta en acciones tales como insultos, ridiculización, humillaciones a través de amenazas verbales, etc. Los autores consideran que la violencia psicológica de control presenta los porcentajes más elevados (entre $90 \%$ y 93,0\%), seguido por la de violencia psicológica de humillación (entre $70,1 \%$ y $72,9 \%$ ). Insultos, gritos, denigración son algunas de las palabras definitorias estipuladas por los estudiantes.
Es de interés investigar sobre el tema, desde un enfoque cualitativo, para de esta forma potenciar relaciones saludables, y contribuir a que los jóvenes universitarios opten por noviazgos no violentos.

El objetivo general es comprender, desde una perspectiva cualitativa, las características de la violencia psicológica en la relación de noviazgo, respecto a sus significados, percepción, modalidades, y respuestas hacia la misma, en estudiantes de inicio de carrera de la Facultad de Psicología de una universidad privada de Mendoza.

\section{Método}

Es una investigación ${ }^{1}$ con enfoque cualitativo ya que se pretende investigar el fenómeno desde una perspectiva personal-subjetiva, lo que permite la comprensión y reconstrucción de procesos psicológicos (Gürtler y Günter, 2007). Su diseño es de tipo fenomenológico, el cual "se enfoca en las experiencias individuales subjetivas de los participantes [...] y se basa en el análisis de discursos y temas específicos, así como en la búsqueda de sus posibles significados" (Mertens, 2005, citado en Hernandez Sampieri et al., 2010, p. 515).

Se trabajó con una muestra intencional, no probabilística, y las unidades de análisis fueron alumnos del $1^{\circ}$ año de la Licenciatura en Psicología y Licenciatura en Niñez, Adolescencia y Familia de la Facultad de Psicología de una universidad privada. La participación fue voluntaria - previa solicitud de consentimiento informado-y se aspiró a conocer percepciones sobre la violencia psicológica en las relaciones de noviazgo en alumnos que inician su carrera.

Participaron un total de 74 estudiantes, en un rango etario de 18 a 21 
años y con una edad media de 19 años, de los cuales 64 fueron mujeres y 10 varones. Se tomaron redes semánticas y se organizaron 11 grupos de discusión del que participaron entre cinco y ocho estudiantes en cada uno. Dos grupos fueron solo de varones y los nueve restantes solo de mujeres.

Se utilizó la triangulación de métodos o técnicas, ya que para recabar información se utilizaron dos técnicas: grupo de discusión y redes semánticas naturales. La estrategia de triangulación permite al investigador una interpretación más acabada del fenómeno en estudio: "La idea subyacente es que al contrastar puntos de vista distintos, teniendo en cuenta el lugar desde donde se enfoca, se obtiene una imagen mucho más completa de un objeto" (Gallart, 1992, citado en Difabio de Anglat, 2005, p. 2).

Las redes semánticas naturales se utilizaron con la frase "Violencia Psicológica" (VP) para analizar los significados atribuidos por los participantes a este fenómeno. Según Figueroa, González y Solís (1981b, citado en Valdez Medina, 1998) esta técnica

(...) tiene como propósito fundamental aproximarse al estudio del significado de manera "natural", es decir, directamente con los individuos, evitando la utilización de taxonomías artificiales creadas por los investigadores para explicar la organización de la información a nivel de memoria semántica, intentando así consolidarse como una de las más sólidas aproximaciones al estudio del significado psicológico, $\mathrm{y}$ con esto, al estudio del conocimiento (p. 65).

A partir de los resultados obtenidos en las Redes Semánticas Naturales, con la palabra estímulo "Violencia Psicológica", se consideró propicio obtener los siguientes puntajes propuestos por Valdez Medina (1998): Valor J, Valor M y FMG. El Valor $\mathrm{J}$ hace referencia a la riqueza semántica de la red; el Valor $M$ total, al peso semántico atribuido a la palabra definitoria de mayor puntaje; y, el valor FMG, a la distancia semántica entre las palabras definidoras, permitiendo observar el nivel de variabilidad en las respuestas aportadas por los participantes.

Valles (2003) enmarca los grupos de discusión dentro de las técnicas cualitativas de conversación. En comparación con las entrevistas individuales en profundidad, los grupos de discusión, en tanto entrevistas grupales, se caracterizan por la concurrencia de varias personas en el encuentro de entrevista. El diseño de los grupos de discusión incluye decisiones muestrales sobre la composición de los grupos (cantidad de participantes), preparativos (contacto previo con los participantes, definición del lugar de reunión, el papel del moderador y de los participantes en el lugar de reunión), así como el registro y tratamiento de la información y el análisis de los datos.

La información aportada por los participantes durante los grupos de discusión fue grabada y luego analizada a través del software Simple Text Analysis ToolTextSTAT versión 2.9c (Hüning, 2000/2014). Es un programa de concordancia que fue diseñado para ser ejecutado fácilmente $\mathrm{y}$ para proporcionar funcionalidad simple. Los textos se pueden combinar para formar las recopilaciones, que se pueden también almacenar como tal. El programa analiza estas 
recopilaciones del texto y exhibe listas de frecuencias de las palabras y concordancias para buscar términos (Escalante Gómez y Páramo, 2011). Permite extraer conclusiones respecto de configuraciones de unidades de significado que se repiten a lo largo de un corpus de datos.

Los grupos de discusión y la administración de redes semánticas se realizaron dentro de la institución participante, en presencia del equipo de investigación. La duración aproximada fue de una hora.

El llegar a establecer un sistema de categorías ajustado a los datos analizados, constituye, de por sí, una vía para obtener conclusiones. Implica una serie de conclusiones acerca de qué tópicos recogen las perspectivas de los estudiantes universitarios acerca de la Violencia Psicológica en el Noviazgo (VPN) y cómo estos temas pueden ser estructurados. Para elaborar tal sistema, se ha planteado el cuestionamiento acerca de los aspectos que comparten unas categorías con otras, en un proceso de comparación intercategorías, que diversos autores han resaltado dentro del análisis de los datos procedentes de grupos de discusión. El hallazgo de elementos comunes, así como los aspectos mencionados por la literatura sobre la temática, han permitido configurar un esquema de líneas temáticas mediante las cuales se agruparon las categorías (ver Anexo 1).

El procedimiento de análisis se centró en la frecuencia de uso de las palabras claves dentro de cada discurso grupal, según las líneas temáticas que guiaron los grupos de discusión y, luego, a través de las concordancias de palabras claves, se realizó un análisis intergrupal de las compartidas por los 11 grupos de estudiantes de $1^{\circ}$ año.
Así, se realizó un proceso de búsqueda de concordancias de unidades de significados entre los grupos de discusión, dentro de la temática de VPN.

A partir de las líneas discursivas básicas desarrolladas y presentadas en el Anexo 1, se ha detectado en el corpus de datos textuales, aquellas palabras clave con mayor frecuencia en la muestra conformada por los discursos de los grupos de $1^{\circ}$ año, obteniendo una lista de estas. Debido a la gran cantidad y diversidad de palabras obtenidas, se estimó oportuno trabajar, dentro de cada línea temática, con las dos palabras clave más importantes de la muestra grupal. Se consideraron palabras claves a aquellas que presentaban mayor frecuencia y que brindaban información de interés para la comprensión de significados sobre la temática en estudio (ver Tabla 1).

$\mathrm{La}$ triangulación de las redes semánticas con el uso de este software permitió la verificación de los resultados obtenidos en los diferentes tipos de análisis de los datos cualitativos.

\section{Resultados}

\section{Resultados de Redes Semánticas Naturales}

A partir de los resultados obtenidos en las Redes Semánticas Naturales de los estudiantes mendocinos, las palabras definitorias de VP, permiten definir este tipo de violencia como una modalidad de maltrato, que involucra la agresión, manipulación, insultos, gritos, celos y negatividad, y puede aparecer la indiferencia. También es conceptualizada como un tipo de abuso, que implica control, dominación y posesión, lo cual provoca dolor, denigración y miedo en quien la padece. 
42 Violencia Psicológica en la relación de noviazgo en estudiantes universitarios que inician...

\section{Tabla 1}

Frecuencias de palabras clave en cada categoría de análisis de los grupos de discusión $(n=11)$

\begin{tabular}{|c|c|c|}
\hline Categoría de análisis & Palabra clave & Frecuencia \\
\hline \multirow[t]{2}{*}{ Aprendizaje a partir de la Experiencia } & es/ser & 13 \\
\hline & no & 14 \\
\hline \multirow[t]{2}{*}{ Cómo afecta la VP en futuras relaciones } & estar & 15 \\
\hline & personas & 8 \\
\hline \multirow[t]{2}{*}{ Cómo afecta la VP en la relación actual } & no & 34 \\
\hline & yo & 28 \\
\hline \multirow[t]{2}{*}{ Consideración de la VP como problema } & montón/mucho/muy & 18 \\
\hline & problema/s & 31 \\
\hline \multirow[t]{2}{*}{ Definición de la VP } & persona/s & 37 \\
\hline & violencia & 19 \\
\hline \multirow[t]{2}{*}{ Diferencias entre VP y V Física } & psicológica/o & 42 \\
\hline & violencia & 47 \\
\hline \multirow{2}{*}{ Diferencias generacionales } & es & 9 \\
\hline & no & 11 \\
\hline \multirow[t]{2}{*}{ Emociones que genera la VP } & estar & 41 \\
\hline & sentir/sentimiento & 23 \\
\hline \multirow[t]{2}{*}{ Experiencia de victimización } & celos & 22 \\
\hline & mucho/muy & 34 \\
\hline \multirow[t]{2}{*}{ Factores que influyen en la VP } & creer & 8 \\
\hline & otro/a/s & 20 \\
\hline \multirow[t]{2}{*}{ Hombre más violento que la mujer } & mujer/es & 9 \\
\hline & no & 11 \\
\hline \multirow[t]{2}{*}{ Manifestación de la VP } & $\cos a / s$ & 13 \\
\hline & estar & 17 \\
\hline \multirow[t]{2}{*}{ Razones para ejercer/sufrir VP } & culpa & 10 \\
\hline & es/ser & 99 \\
\hline \multirow[t]{2}{*}{ Reacciones frente a la VP } & no & 58 \\
\hline & yo & 27 \\
\hline \multirow[t]{2}{*}{ Recursos de ayuda } & poder & 5 \\
\hline & yo & 8 \\
\hline \multirow[t]{2}{*}{ Síntesis/reflexión final } & es/ser & 29 \\
\hline & poder & 9 \\
\hline \multirow[t]{2}{*}{ VP más dañina que la VF } & es/ser & 39 \\
\hline & psicológico/a & 22 \\
\hline
\end{tabular}


A partir de la palabra estímulo VP se obtuvieron los siguientes puntajes. El Valor $\mathrm{J}$, que hace referencia a la riqueza semántica de la red, lo constituye un total de 137 palabras definidoras. En cuanto al Valor $\mathrm{M}$ total, ${ }^{2}$ que hace referencia al peso semántico atribuido a la palabra definitoria de mayor puntaje, cabe destacar que la palabra clave de mayor puntaje fue "maltrato" y recibió una puntuación de 261 (Tabla 2).

De la misma manera, teniendo en cuenta el valor FMG, que permite observar la variabilidad en las respuestas dadas por la distancia semántica entre las palabras definidoras, se observa que el núcleo semántico presenta consistencia entre los alumnos en las primeras tres palabras (maltrato, agresión y manipulación), pero existe una diferencia en los puntajes con respecto a las palabras que le siguen, lo que da cuenta de una gran variabilidad en las respuestas aportadas por los estudiantes que inician la carrera.

En la Tabla 2 y gráfico 1 se puede observar que los alumnos de $1^{\circ}$ año definen la VP, como un tipo de maltrato $(\mathrm{VM}=261)$ que involucra la agresión $(\mathrm{VM}=217)$ y la manipulación $(\mathrm{VM}=214)$, como principales características. Así mismo, la VP se encuentra identificada a través de conductas, que le son propias, tales como: insultos $(\mathrm{VM}=$ $188)$, gritos $(\mathrm{VM}=92)$, celos $(\mathrm{VM}=81)$, negatividad ( $\mathrm{VM}=68)$ y control $(\mathrm{VM}=66)$. A estas palabras se agregan otras que también integran el significado de VP como: denigración $(\mathrm{VM}=60)$, posesión $(\mathrm{VM}=$ 54), indiferencia $(\mathrm{VM}=54)$, dominación $(\mathrm{VM}=48)$ y abuso $(\mathrm{VM}=48)$. Por otro lado, produce consecuencias de tipo psicológico $(\mathrm{VM}=65)$ asociadas con el dolor $(\mathrm{VM}=65)$ y el miedo $(\mathrm{VM}=58)$.

\section{Resultados del análisis de los grupos discusión}

\section{Figura 1}

Valor $M$ de las palabras definidoras de los estudiantes de primer año $(n=74)$

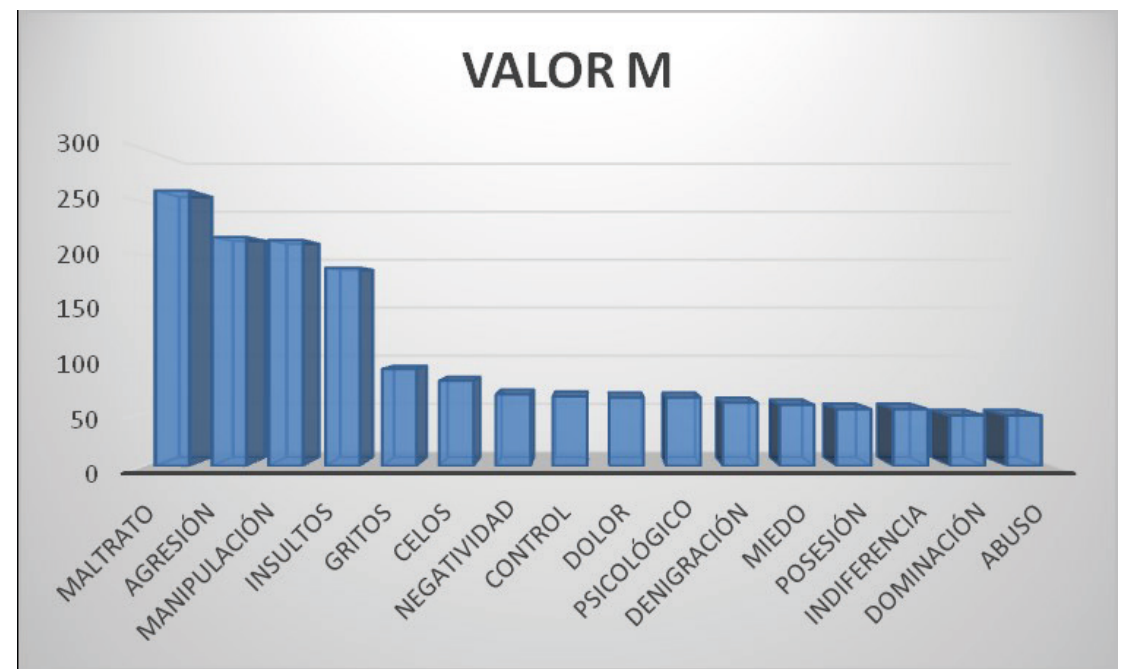


44 Violencia Psicológica en la relación de noviazgo en estudiantes universitarios que inician...

Tabla 2

Conjunto SAM de los estudiantes de primer año $(\mathrm{n}=74)$

\begin{tabular}{lll}
\hline Palabras definidoras & Valor M & Valor FMG \\
\hline Maltrato & 261 & $100 \%$ \\
Agresión & 217 & $83 \%$ \\
Manipulación & 214 & $82 \%$ \\
Insultos & 188 & $72 \%$ \\
Gritos & 92 & $35 \%$ \\
Celos & 81 & $31 \%$ \\
Negatividad & 68 & $26 \%$ \\
Control & 66 & $25 \%$ \\
Dolor & 65 & $24 \%$ \\
Psicológico & 65 & $24 \%$ \\
Denigración & 60 & $23 \%$ \\
Miedo & 58 & $22 \%$ \\
Posesión & 54 & $20 \%$ \\
Indiferencia & 54 & $20 \%$ \\
Dominación & 48 & $18 \%$ \\
Abuso & 48 & $18 \%$ \\
\hline
\end{tabular}

Como ya fuera anticipado, para describir los significados y la percepción de la VP en los integrantes de la pareja de novios (datos obtenidos del análisis en TextSTAT de los grupos de discusión), cada palabra clave fue contextualizada analizando las concordancias arrojadas por el TextSTAT y destacando los significados semejantes.

En los grupos de discusión se consideraron las dos palabras clave más significativas y frecuentes en cada categoría de análisis, algunas resultaron ser las palabras claves en más de una categoría de análisis. Las palabras claves fueron: "ser", "no", “estar”, “yo", "persona”, "poder”, "mucho”, "violencia", "psicológica", "problema", “sentir", “celos", “culpa", “cosa”, “creer", "otro" y "mujer".

Las categorías de análisis

surgidas de los grupos de discusión son las siguientes:

\section{$1^{\circ}$ La definición de violencia psicológica}

Las palabras clave más significativas son "persona" y "violencia". Así, la VP aparece ligada a las personas, es decir, a quien la ejerce y a quien la padece. Señalan "es deshumanizar en cierta parte a la otra persona”, ya que en ella está presente la manipulación, el poder, la dominación y el menosprecio.

En las concordancias de la palabra 
"violencia" los estudiantes se enfocan en el daño y el dolor que produce: "es un daño más que nada con palabras". Entienden que una de las características de este tipo de violencia es la utilización del lenguaje como instrumento para ejercerla.

\section{$2^{o}$ Las diferencias entre la violencia psicológica y la violencia física}

En esta línea temática se han seleccionado dos palabras claves "violencia" y "psicológica". Los participantes consideran como distintivo de la VP que esta es "es menos evidente", por lo que la principal diferencia con la física es que no deja marcas perceptibles a simple vista. Coinciden en que la violencia psicológica es "más constante" y que antecede a la física "primero se da la violencia psicológica y después se da la física". Concluyen que violencia física presupone a la psicológica.

La palabra clave "psicológica", aparece vinculada a la gravedad: "es mucho peor la psicológica", debido a su persistencia, a que puede pasar desapercibida, a sus múltiples consecuencias nocivas, entre otras razones. El carácter silencioso de la violencia psicológica permite que pueda ejercerse en diferentes ámbitos (pareja, trabajo).

\section{$3^{\circ}$ Violencia psicológica más dañina que la violencia física}

En esta línea temática las palabras clave a destacar son "es/ser" y "psicológico/a".

Coinciden en que la VP es más dañina que la física por diversos motivos: "la diferencia es que puede durar más, es como más constante", "es como que no podés distinguir si lo que te dicen es verdad", "es más difícil que alguien pida ayuda... después es más difícil darse cuenta que era violencia lo que te estaba haciendo", "es más difícil recuperarse", "para mí es más complejo porque pasa desapercibido, a lo físico lo ves, en cambio lo psicológico no te das cuenta... es progresivo", "es más profunda, es más dolorosa en muchos sentidos, ataca lo que es la persona en sí". Pero también consideraron que ambos tipos de violencia se dan casi simultáneamente: “...para mi van acompañadas... es un antes y un después... no hay una sin la otra", "la violencia física afecta lo psicológico también”.

En consonancia con lo anterior, se alude a la gravedad y daño de la psicológica: "la psicológica, porque si bien la física te deja marcas, como por ejemplo, cicatrices o machucones, vos te curas eso y se te pasa, en cambio la psicológica te deja una marca más fuerte", "lo físico no le genera nada más que las repercusiones del cuerpo mientras que en cualquier violencia psicológica entra a un estado de angustia extrema o ganas de hacerse daño, hasta la autoflagelación". Entienden que la VP va generalmente acompañada de la física: "yo creo que la violencia física lleva a la psicológica", "claro, yo pienso que la violencia física como que va de la mano con la psicológica. Por más de que te estén pegando nada más, ya te están matando por adentro también".

\section{$4^{o}$ Factores que influyen en la violencia psicológica}

Las palabras clave que se destacaron fueron: "creer" y "otro/a/s".

Utilizan la palabra "creer" para describir cómo el/la victimario/a actúa sobre el/la víctima: "lo manipula para que termine creyendo que es culpa de la otra persona". Entre los factores que influyen en la VP mencionan: "el aprendizaje familiar", "haber tenido padres agresivos", "inseguridad de 
la persona", "los aspectos históricos (antes estaba permitido cierta dominación del hombre hacia la mujer)".

En cuanto a la palabra "otro/a" se consideró que, a veces, se mantiene una relación violenta por miedo a perderlo: "que uno tenga miedo de perder al otro". Se aludió a la inseguridad de quien ejerce la violencia, manipula para sentir que ejerce el control. Se destacó la idea de que en otras culturas este tipo de relación violenta está naturalizada.

$5^{\circ}$ Manifestación de la violencia psicológica

Las palabras clave que se destacaron en esta línea temática han sido "cosa/s" y "estar".

Señalan muchas cosas que reflejaban la VP: "empezando por separarla de sus amistades o de otros vínculos, cosa de ser él su único apoyo, a quien recurrir", "que siempre éramos de salir juntos, de tomar juntos y se volvía loco, capaz que era una mínima cosa y se volvía loco, y me agarraba, me apretaba y él después me pedía perdón", "debilitamiento de la otra persona, haciéndola sentir como inferior o recalcándole todo el tiempo sus cosas malas o metiéndole ideas falsas en la cabeza". Por otro lado, el hecho de haber vivido la experiencia les permite detectar ciertas "cosas", y estar en alerta para que no vuelvan a ocurrir: "a partir de haberlas vivido, yo sé qué cosas sí y que no, ahora ya estoy tranquila pero sé que la primera cosa que no me guste de la persona, que estoy ahora, sé cómo tengo que reaccionar, son cosas que no me las voy a permitir nunca más, que me ha servido".

En cuanto a la palabra "estar", recordaron lo que les ocurría cuando estaban en una relación violenta: "para que no se enoje, y así fue como [...] terminaba sin salir o sin ir a bailar, sin estar con amigas, sin ponerme la ropa que quería, pensando que lo que hacía estaba bien", "porque te deja maquinando todo el tiempo: "hice algo mal, estoy fea todo el tiempo', estar pendiente en esa persona". Y para señalar que, finalizada la relación, las persona logra "estar más tranquila".

\section{$6^{o}$ Consecuencias emocionales de la violencia psicológica}

Al analizar las palabras claves de esta línea temática, se observa que las consecuencias emocionales más significativas de la VP aparecen relacionadas al "estar" y al "sentir".

En relación a la palabra clave "estar" el grupo da cuenta de cómo la violencia atraviesa el sí mismo. Al compartir su propia experiencia de victimización, una estudiante refiere "está muy lejos del amor cuando una persona te infringe ese tipo de cosas, estas más cerca de su egoísmo que otra cosa y está muy cercano al desprecio que nos tenemos a sí mismos". Sumado a este sentimiento de desprecio y negación de uno mismo, otra de las participantes detalla "más que nada angustia, estar todo el tiempo con ese sentimiento de culpa".

La palabra "sentir" se encuentra acompañada de variados significados negativos producidos por la VP. Una alumna detalla su vivencia "me sentí como objeto de manipulación". Describe cómo esta violencia afecta el bienestar de la persona: "el sentirse vulnerable", "inútil", "insuficiente", "inferior", "impotencia".

\section{$7^{o}$ Razones para ejercer/sufrir violencia psicológica}

En esta línea temática las palabras 
clave a destacar son "culpa" y "es/ser".

La palabra "culpa" alude a cómo el/ la victimario/a hace sentir a su víctima: "y él se victimizaba, y lo que me pasaba es que me generaba culpa, entonces yo volvía a él, pidiéndole perdón a él, aunque yo no tuviera la culpa", "me hacía sentir culpable de cosas que en realidad yo nunca había hecho".

Con el verbo "ser" se caracteriza la relación violenta "era una relación anormal", "era como si fuese un chiste"; "intentamos cambiar pero es como que siempre volvemos a caer en esas cosas", "pero lo que pasa es que yo también, en parte, le recrimino, o le reprocho cosas, como que es mutuo en este caso, entonces es como que es más difícil". Se alude a las vivencias en el seno familiar "el círculo familiar es con lo que empezás a tener autoestima" y lo aprendido en él se extiende a otras relaciones amorosas. También utilizan el término para aludir al sufrimiento experimentado: "es como estar enfermo y no saberlo, es algo que lo llevás, como el dolor de muelas, a alguien le duele la muela puede parecer que está bien, pero por dentro se está matando y es algo que te punza, sabes que molesta pero lo seguís tapando".

\section{$8^{o}$ Reacciones frente a la violencia psicológica}

Con mayor frecuencia se presentaron las palabras clave "no" y "yo".

El "no" expresa todo lo que no hacían frente a la ocurrencia de un episodio de VP: "no nos hablábamos más", "no pedía perdón", "no se dan cuenta", "no te dicen nada porque no les importa"; las prohibiciones a las que eran sometidas: "no podía salir con mis amigas, no me tenía que juntar con ellas"; para aludir a la descalificación que sufrían y lo que esto les generaba: "él me decía que yo no servía para nada y capaz que él lo decía más como una joda, o no sé, y yo no me lo tomaba así... pero a mí no me gustaba ese trato". También expresaron la dificultad para ayudar a quien sufría VP ya que muchas veces "no se daban cuenta" que estaban en una relación violenta: "para que se separara, de esa situación y ella se diera cuenta, pero después volvió a caer y ahora ya no tenemos como influencia en ella, como que se ha separado de nosotras, él lo logró".

La palabra "yo" es utilizada para referir vivencia de victimización en primera persona: "yo esperaba que cuando él se equivocara, me pidiera perdón [] yo terminaba y volvíamos, terminaba y volvíamos, se volvió algo repetitivo [] yo respondía con sumisión [] yo me alejaba de mis amistades [] yo decía 'no pasa nada, es normal"".

\section{$9^{\circ}$ Experiencia de victimización en las/los participantes}

Las palabras clave "celos" y "mucho/muy" fueron las de mayor frecuencia y significatividad en esta línea temática.

Los "celos" son reconocidos como algo propio y también como algo padecido. Una alumna manifiesta "detecté la violencia psicológica a través de los celos, el control, de compartir la relación a través de las redes sociales..." y un alumno señala: "[...] yo me voy a juntar con mis amigas y ella tenía celos a todas mis amigas, ¿verdad? y son mis amigas, no más". También los reconocen como propios "hace un tiempo corté porque yo era muy celosa y muy posesiva, él salía y yo estaba como ¿qué vas a hacer? ¿Con quién vas a salir?"; "imaginate cuando corté 
(porque yo decidí cortarle a ella), me entero por ella que los que yo les tenía celos, le empezaron a tirar onda".

Las palabras "muy/mucho", aluden a las consecuencias negativas producidas por la experiencia de victimización, tales como "tener mucha inseguridad", "no confiar mucho en la gente". También para caracterizar la relación: "había mucha manipulación de por medio, mucha dependencia de mí", "mucha manipulación y mucha inseguridad de mi parte". Se observan justificaciones de la VP padecida (debido a la edad o a ciertas características personales): "no es que lo justifique pero era muy chica, que tenía muy baja autoestima, me dejaba manipular y cosas así, caí en depresión". Se enfatiza la naturalización de conductas que expresan VP: "la persona lo tenía muy naturalizado, yo cuando le decía "no me grités', era como ‘¿cómo no te voy a gritar? Si es algo sumamente común"”.

\section{$10^{\circ}$ Aprendizaje a partir de la experiencia de victimización de las/los participantes}

En el discurso de los alumnos ${ }^{3}$ las palabras claves sobre el aprendizaje a raíz de la experiencia de la violencia, corresponden a "es/era" y "no". En cuanto a las palabras claves "es y era", las mismas se pueden articular en una unidad de significado "Ser". Estos códigos hacen referencia a las enseñanzas que puede dejar la experiencia de la violencia psicológica. Entre ellas, las mujeres mencionan la posibilidad de detectar en otras relaciones los signos de la VP: "es como que te podes dar cuenta", considerando volverse más precavidas y capaces de poner límites.

Una de las palabras que más se repite es "no", vinculada directamente con el límite. Una alumna lo relaciona con el poder discriminar entre "estas cosas me las merezco, estas no". Es precisamente este límite el que capitalizan como aprendizaje para no repetir la experiencia de victimización. También aparecen ligados a los continuos "no" que reconocen haber recibido de sus parejas lo que las dañaba.

\section{$11^{\circ}$ Consecuencias de la violencia psicológica en la relación actual}

Las palabras clave son "no" y "yo". El "no", aparece vinculado, por un lado, con la negación de uno mismo como consecuencia del vínculo violento, y, por otro lado, en relación a la nueva pareja: así, por ejemplo, una alumna destaca "sentía que era todo mentira, no le creía" y un varón "no me he puesto de novio porque le tengo manso miedo a los cuernos".

Las experiencias previas parecen haber generado condicionamientos que impiden que la persona asocie el estar en pareja como algo que pueda resultar nutricio, una experiencia de amor, respeto y confianza. Se observa que esta desconfianza se extiende a otras relaciones (familia, amigos) "te cambia, ya no sos la de antes, te da marcas y es difícil salir adelante después de eso [...] para mí no solo con la relación que vas a tener con la otra persona, sino también con amigos o con la familia".

En cuanto a la palabra "yo", esta alude al "sí mismo" que se ve afectado aun luego de haber terminado la relación violenta. Se enfatiza la persistencia del daño sufrido en cuanto continúa presente más allá del fin de la relación: una alumna refiere "me afectó en eso de que no era feliz y no estaba siendo yo misma". 
$12^{\circ}$ Consecuencias de la violencia psicológica en futuras relaciones

Las palabras claves son "Persona" y "Estar". "Persona" hace referencia, por un lado, a quien ejerció violencia en una relación pasada, y, por el otro lado, a la persona con la que se formará una nueva relación. Al respecto, dan cuenta de que requieren aprender a vincularse con esta nueva persona, dejando atrás sus experiencias del pasado.

"Estar" da cuenta de la influencia que tiene quien ejerce VP en una relación, así, una estudiante señala "yo estaba bien después de todo y volví, sabía que estaba mal pero igual me volví a confundir”.

\section{$13^{\circ}$ Recursos de ayuda}

En cuanto a esta línea temática, se presentaron con mayor frecuencia las palabras clave "poder" y "yo".

Mencionan la importancia de un psicólogo para "poder descubrir lo que está pasando y poder salir de una relación violenta". Algunas participantes refieren que al intentar poner fin a la relación ellas pensaban " "no puedo estar sin él, no puedo estar sin él' y cuando él me hizo ese chiste de que me iba a dejar casi me muero".

Con la palabra "yo" se refirieron a la inmadurez de las primeras relaciones como una posible causa del padecimiento de VP: "yo era muy chica, por eso lo sufrí", pero también el darse cuenta de que se puede seguir viviendo de otra forma: "cuando estaba muy enferma me di cuenta que no valía la pena, que yo no necesitaba a esa persona... yo ya no necesitaba eso, no quería eso para mí".

\section{$14^{\circ}$ La violencia psicológica como problema}

Dentro de esta línea temática, se destacan las palabras claves "problema/s" y "mucho". Esta última incluye otros códigos como "montón, muchos, muy, muchísimo". Estas palabras aluden a todo aquello que se ve afectado por la VP: una alumna refiere "te arruina un montón de cosas", sus consecuencias son personales — "autoestima"_ y alcanzan otras relaciones —_...y como decía ella que le hacían bullying, eso también puede ser una violencia social, pasa muchísimo...". También alude a la naturalización de la violencia "que además esta normalizado y muy naturalizado".

Cuando se menciona la palabra clave "problema", los estudiantes consideran la VP como "un problema tanto cultural como social". Se engloba a la VP como un problema que abarca distintos planos: "social, cultural, psicológico, emocional". Sin embargo, enfatizan su cualidad multidimensional, no existiendo consenso sobre su consideración como problema social o de pareja.

\section{$15^{\circ}$ Diferencias generacionales al considerar la violencia psicológica}

Dentro de esta categoría se encuentran las palabras claves "ser" y "no". La primera incluye las conjugaciones del verbo "eran, es", para dar cuenta de las diferencias en las concepciones de la violencia "antes y ahora". Al respecto, una de las estudiantes destaca "antes se naturalizaba más, o era como que se aguantaba, había más tolerancia". En este sentido, se percibe la VP como un constructo actual, debido a que ciertas conductas hoy consideradas como expresión de VPN, antes no eran percibidas como tal.

La palabra "no" sirve para enfatizar que lo que hoy puede considerarse como 
violencia antes "no lo era". Un estudiante admite "en las generaciones anteriores no se daban cuenta de lo que era violencia psicológica en sí". Por otro lado, la palabra "no" alude a cómo era la mujer antes y todo aquello que no le era permitido: "no se defendía", "no poder trabajar".

\section{$16^{\circ}$ Hombre más violento que la mujer}

Con mayor frecuencia se observaron las palabras "mujer/es" y "no".

Mencionan ciertos permisos sociales hacia el hombre que expresan violencia hacia la "mujer": "el concepto de acostarse con muchas personas también es distinto, porque la mujer que se acuesta con muchas personas es una puta, en cambio en los hombres, es un capo".

En relación a la palabra "no", la misma se utiliza para hacer referencia al "no poder" de las mujeres en comparación con el hombre "mi ex siempre me decía 'porque vos tenés toda una vida sexual pasada y porque yo no, y porque en mi casa me dijeron que las mujeres no pueden". No obstante, enfatizan el hecho de que las mujeres no están exentas de ejercer violencia, es decir que tanto mujeres como varones pueden ser activos en el ejercicio de la VP.

\section{$17^{\circ}$ Síntesis/reflexión final}

Como síntesis o reflexión de la discusión grupal, las palabras "es/ser" y "poder" fueron las más elegidas por los participantes.

Los aportes del grupo pueden sintetizarse de la siguiente manera: "es necesario saber qué es, qué consecuencias tiene, en qué se ve implicado y en qué se puede diferenciar...es un patrón de conducta que tiene una persona agresiva y lo que hace es dirigir esa conducta violenta sin tener en cuenta sus fines ... y consecuencias que trae, lo que es el aislamiento por parte del sumiso porque está sometido, tiene repercusiones a nivel psicológico y ... a nivel emocional ... a nivel cultural y a nivel individual".

Añaden que es bastante normal caer en ese tipo de relaciones enfermizas: "tiene un carácter estructurante y que iba a influir en nuestra visión de las relaciones, las que vamos a establecer, las posteriores..." por lo tanto, que es necesario "dejar de pensar que el otro es un objeto al que podés decirle que hacer, y cómo vestirse, y todas esas cosas...". Se enfatiza la importancia del empoderamiento de la persona "...apoyar a esa gente... es la única manera en que ni el novio, ni el jefe, ni la profe la maltrate", enfatizando aspectos vinculados a la promoción de la salud: "fomentar lo positivo, así no dejas que te lo hagan y tampoco hacerlo".

En cuanto a la palabra "poder", se insistió en que la VP "es muy difícil reconocerla y poder actuar ante ella", pero que se puede superar con el apoyo de familiares y profesionales.

\section{Discusión}

\section{Redes semánticas naturales}

En coincidencia con los resultados del presente estudio, diversas investigaciones resaltan la prevalencia de la VPN. Calderón Guerrero (2019) afirma que, en las relaciones de noviazgo, predominan la violencia psicológica y la verbal-emotiva frente a los otros tipos de violencia.

Del conjunto SAM, que muestra las palabras definitorias de VP, se desprende que ésta es considerada principalmente como un tipo de maltrato, que involucra la agresión y 
la manipulación.

La Organización Mundial de la Salud (2003) considera que la violencia de pareja involucra comportamientos que causan daños físicos, sexuales o psicológicos, tales como la agresión, la coerción sexual y el maltrato psicológico. En consonancia con esto, el maltrato constituye la palabra que mejor define a la VP para los participantes del presente estudio.

Velázquez Rivera (2011) sostiene que, en una relación de noviazgo, la fuerza física o manipulación psicológica, puede utilizarse para controlar al otro integrante de la pareja. La manipulación constituye una de las palabras del núcleo semántico de VP.

Si bien los insultos y gritos fueron reconocidos como manifestación de la VP por el $76 \%$ de los participantes en una investigación de Oviedo Aguilar (2017), el autor considera alarmante que no sean reconocidos como tales por la totalidad de la población estudiada, lo que permite concluir que 1 de cada 4 participantes "naturaliza" el trato violento en las relaciones de pareja.

Una de las palabras definitorias en las redes semánticas fue celos. Marcos Santiago e Isidro de Pedro (2019) revelan lo frecuente de los comentarios y bromas sarcásticos de carácter celotípico hacia las mujeres por parte de sus parejas y las acusaciones de infidelidad o de coquetear con otros. En concordancia con esto, del análisis de los grupos de discusión, se detecta la siguiente frase de un participante varón "cuando corté (porque yo decidí cortarle a ella) me entero por ella que los que yo les tenía celos, le empezaron a tirar onda, entonces yo ahí me di cuenta de que no estaba tan equivocado yo".
A modo de síntesis, de los resultados obtenidos se desprende que la VP es considerada como un tipo de maltrato que implica daño a la otra persona a través de diversas conductas que causan efectos devastadores en el psiquismo de la víctima generando experiencias de dolor y miedo.

\section{Grupos de discusión}

Si bien se ha reportado que uno de cada cinco estudiantes secundarias mujeres reconocen haber sufrido abuso físico y/o sexual por parte de su pareja (APA, 2015), Cantor et al. (2020) consideran que son los alumnos universitarios los que tienen mayor riesgo de perpetrar y padecer violencia en el noviazgo.

Asociado a las Consecuencias de la $V P$, los estudiantes reconocen cómo la VP padecida puede afectar las futuras relaciones de pareja de la persona, malestar que puede extenderse a otros vínculos - familiares $\mathrm{y}$ amigos. Al respecto, en un estudio de Rodríguez Hernández et al. (2018) indagaron sobre la violencia y el apoyo social percibido en jóvenes universitarios españoles, y sostienen que, aún cuando las personas cuenten con apoyo suficiente de familia y amigos, éste puede no ser efectivo para enfrentar la violencia, debido a su falta de adecuación a la situación o que esté acompañado por críticas o comentarios donde se les culpabilice por la situación. Los estudiantes mendocinos destacan, como principales sentimientos frente a la VP, la culpa y la desvalorización. Blázquez Alonso et al. (2015) señalan que los estudiantes reportaron conductas de desvalorización y culpabilización en sus relaciones de pareja, lo que coincide con los hallazgos de la presente investigación.

Una consideración especial merece 
el tema de la autoestima. Los participantes consideran que la VP daña la autoestima de la víctima. Castillo Saavedra et al. (2018) investigaron la relación entre la violencia de género y la autoestima. Se evidenció que el $52,8 \%$ de las mujeres víctimas de violencia de género presentaron una autoestima baja. Consideran que las consecuencias de tal tipo de violencia pueden ser devastadoras, incrementando las posibilidades de presentar algún padecimiento mental, tales como estados depresivos o suicidio en los casos más graves. Esto es mencionado por los participantes en relación a dos categorías, por un lado relacionado con VP como problema, considerándola como un problema grave debido a sus múltiples consecuencias en el psiquismo de la víctima; $y$, por otro lado, a Factores que influyen en la VP, aludiendo a la dinámica vincular establecida entre víctima y victimario: un miembro de la pareja necesita manipular y controlar, y, el otro, lo permite, entre otros factores, debido a su baja autoestima.

Escoto Sainz et al. (2007) reconocen la VPN como una problemática actual. Comparan sus secuelas con las de la violencia física y al respecto señalan: "éstas [heridas físicas] se pueden borrar, en cambio, los recuerdos derivados de la violencia psicológica son más difíciles de olvidar" (p.30). Los participantes destacan la persistencia de las consecuencias de la violencia psicológica a través del tiempo, aun cuando la relación abusiva hubiera terminado.

En la categoría Manifestación de la $V P$ los estudiantes mendocinos destacaron cómo el victimario producía debilitamiento de la otra persona, haciéndola sentir como inferior o recalcándole todo el tiempo sus cosas malas. En consonancia con esto, el equipo guatemalteco (Escoto Sainz et al., 2007) destacaba que el $70 \%$ de las jóvenes encuestadas reconocen haber sido criticadas por sus parejas por su forma de ser y el $56.7 \%$ haber recibido burlas en la relación. Los estudiantes mendocinos refieren haberse sentido "inútiles o inferiores" en su pareja, en consonancia también con lo detallado por Castillo Saavedra et al. (2018). L a culpa es concebida también como una de las Razones para ejercer/sufrir VP, enfatizando que quien ejerce violencia hace sentir culpa a la víctima.

Los adolescentes identifican diferentes patrones de violencia, tales como la violencia psicológica, sexista o verbal (Pérez-Marco et al, 2020). En relación a la frecuencia de la violencia psicológica en el noviazgo, un estudio con jóvenes mexicanos (Peña Cárdenas et al., 2013) halló que el $46.2 \%$ del grupo total sufre de violencia en alguna medida. Sin embargo, las conductas predominantes se refieren a las discusiones en la pareja, el aislamiento del grupo de amigos y la invasión de privacidad. No obstante, una de las principales diferencias con este estudio, es que, si bien los jóvenes mejicanos no conciben la manipulación como rasgo predominante en los vínculos en los que se da la VP, los estudiantes mendocinos destacan la manipulación como palabra definitoria de la violencia psicológica en la pareja.

En la categoría La VP como problema los estudiantes enfatizan su consideración en tanto problema multidimensional. Esta consideración difiere de los hallazgos de una investigación anterior en el cual la VP es considerada, en mayor medida, como un problema de pareja y, en menor medida, como un problema social 
(Páramo y Arrigoni, 2018).

En cuanto a la propia Experiencia de victimización, los estudiantes mendocinos reconocieron haber sufrido celos enfermizos, por parte de la pareja o haberlos experimentado ellos mismos, cuando eran muy jóvenes. Estos hallazgos coinciden con lo de Rojas Solís (2013) quien afirma que la violencia en el noviazgo puede ser cuantitativa y cualitativamente diferente de aquellas ejercidas en edades adultas, por lo que las actitudes y creencias sexistas y los celos "románticos" son la base de la violencia que ocurre en relaciones de pareja a edades tempranas (Rodríguez Domínguez et al, 2018).

En cuanto a si el Hombre es más violento que la mujer, se observó el desacuerdo de las estudiantes con ciertos tratos diferenciales que hacen que la sociedad permita al hombre ciertas cosas vedadas o consideradas desde una diferente perspectiva en relación a las mujeres, esto vinculado al sexismo denunciado por diversos autores (García Díaz et al, 2020; Rodríguez Hernández et al, 2018; Vázquez García y Castro, 2008), presente aun en la sociedad actual. De todas formas, en consonancia con Toldos Romero (2013), los estudiantes mendocinos reconocen que los hombres también pueden ser víctimas de mujeres agresoras. Benavides Delgado (2016) en su estudio evidenció la existencia de diferentes violencias en el noviazgo, y pudo determinar que tanto hombres como mujeres se sentían víctimas $\mathrm{y}$ agresores, desmitificando el noviazgo como una etapa idílica, por un lado, y cuestionando los roles tradicionales, en los que se sitúan los hombres como agresores y las mujeres como víctimas, por otro lado.

El maltrato constituye la palabra que mejor define a la VP de acuerdo a los participantes del presente estudio. A modo de Síntesis/Reflexión final coinciden en que la VP es un tipo de maltrato, del orden de lo psicológico, que implica la agresión de una persona a otra, a través de conductas manipuladoras, y controladoras, que causan efectos nocivos en quien la padece.

\section{Conclusiones}

Los estudios cualitativos se caracterizan por ser un proceso en espiral, es decir que, una vez en contacto con el campo se comienza a recabar información a través de las diferentes técnicas seleccionadas, y se logra cumplimentar los objetivos de la investigación alcanzando la saturación teórica de significados.

La violencia en las relaciones de noviazgo ha sido estudiada desde hace ya varios años. Así, White \& Koss (1991) reportaron que este tipo de violencia afectaba al 39\% de los estudiantes universitarios. En el año 2001 Silverman et al. (2001) hallaron que el $10 \%$ de los estudiantes secundarios padecían violencia - física y sexual- en el noviazgo. Una reciente investigación presentada en la 121 Convención Anual de la American Psychological Association en Estados Unidos, se informa que 1 de cada 3 jóvenes entre 14 y 20 años de edad, reconoce haber sido víctima de violencia en el noviazgo, y, además, casi 1 de cada 3 reconoce haber cometido violencia contra su pareja (APA, 2020). Estos datos reflejan lo extendido de la problemática.

Del análisis de las Redes Semánticas, se pudo apreciar que los estudiantes universitarios conciben la VPN como forma de maltrato que socaba el 
psiquismo de la víctima, por lo que recalcan la importancia de instrumentar, por un lado, acciones de prevención para que los jóvenes puedan "darse cuenta" o identificar las señales de este tipo de violencia; $y$, por el otro lado, de atención a víctimas y victimarios: "Hay que aceptar y apoyar a las personas que se encuentran en esa situación", "Ayudar a acrecentar el autoestima... sobre todo eso que dijiste de acrecentar el autoestima, porque hay momentos en los que como que ya es tarde y no hay manera, entonces hay que empezar desde casa y desde muy chiquitas o entre nosotras", "Atención psicológica, indudablemente en la mayoría de los casos la mujer que está sufriendo violencia necesita de un psicólogo porque no pueden salir".

Del análisis de los discursos grupales obtenidos en los grupos de discusión, a través del TextSTAT se han hallado significados que coinciden, en general, con los hallazgos de las investigaciones de los últimos 10 años sobre la temática en cuestión.

Todas las líneas temáticas y categorías construidas por este equipo de investigación han sido representadas claramente en el discurso de los estudiantes. En la última categoría, Síntesis/Reflexión final, los participantes remarcaron la importancia de comprender y ayudar a las personas que se encuentran en esta situación, de la necesidad de brindar apoyo familiar y social, reforzar la autoestima, del diseño de programas de prevención de la violencia en el noviazgo desde edades tempranas, y de consultar a profesionales de la salud cuando sea pertinente. Esto se vislumbra en los ejemplos aportados anteriormente sobre la atención de víctimas y victimarios.
Debido a que investigaciones recientes con estudiantes universitarios reportan que, "la tolerancia a la violencia en la pareja y a las actitudes sexistas fueron mayores, especialmente entre estudiantes de psicología" (García-Díaz et al, 2020, p. 179), se estima propicia la instrumentación de programas específicos de promoción de la salud en general y de prevención de la problemática de la violencia relacional en particular, desde las universidades. Asimismo, se considera propicio incluir en los planes de formación para futuros psicólogos espacios curriculares que brinden herramientas para la detección y abordaje temprano y eficaz de este fenómeno, con el fin de evitar graves daños en el psiquismo de las personas, debido a la naturaleza persistente, dañina y silenciosa de la VP.

En virtud de que la VPN es un problema que reviste cierta gravedad, se estima conveniente ampliar este tipo de investigaciones en otros estudiantes locales y nacionales. El hecho de que los participantes conciban a la VPN como un problema multicausal que lleva a naturalizar los malos tratos en la relación vincular, es un aliciente para continuar investigando la temática en futuras líneas de investigación.

Como limitaciones encontramos que, por las características de las carreras que estudian los participantes, los varones han estado representados en menor cuantía que las mujeres. Por esto, se estima de interés desarrollar estudios similares en facultades con alta prevalencia de varones, y así realizar estudios comparativos, lo que podría concretarse en futuras líneas de investigación. 


\section{Referencias}

Alegría del Ángel, M. \& Rodríguez Barraza, A. (2015). Violencia en el noviazgo: perpetración, victimización $\mathrm{y}$ violencia mutua. Una revisión. Actualidades en Psicología, 29(118), 57-72. DOI: http://dx.doi. org/10.15517/ap.v29i118.16008

American Psychological Association (2015). Violencia contra la pareja. Recuperado (25 de julio de 2020) de: https://www.apa. org/topics/violence/violenciapareja\#: : text $=\mathrm{M} \% \mathrm{C} 3 \% \mathrm{~A} 1 \mathrm{~s} \% 20$ d e $\% 20 \mathrm{un} \mathrm{a} \% 20 \mathrm{de} \% 20$ cada, $\% 2 \mathrm{C} \% 20 \mathrm{o} \% 20$ novio $\% 2 \mathrm{Fa}$

American Psychological Association (2020). One in Three U.S. Youths Report Being Victims of Dating Violence. Recuperado (25 de Julio 2020)de: https://www.apa.org/news/press/ releases/2013/07/dating-violence

Benavides Delgado, J. (2016). Violencia en el noviazgo: diferencias de género. Informes Psicológicos, 16(2), 2736. Doi: http://dx.doi.org/10.18566/ infpsicv16n2a02

Blázquez Alonso, M.; Moreno Manso, J.M. y García-Baamonde, M.E. (Marzo 2015). Maltrato psicológico en las relaciones de pareja. Boletín de Psicología, 113, 29-47. Recuperado de: https://www.uv.es/seoane/ boletin/previos/N113-2.pdf

Calderón Guerrero, R.A. (2019). Violencia en el noviazgo, una revisión teórica. (Tesis de Bachiller en Psicología). Recuperado de: http://repositorio. uss.edu.pe/bitstream/handle/ uss $/ 6099 /$ Calder $\%$ C3\%B3n $\% 20$
Guerrero\%20Rosa\%20Anshela. pdf? sequence $=1$ \&isAllowed $=y$

Cantor, N., Joppa, M., Angelone, D. (2020). An Examination of Dating Violence Among College Student-Athletes. Journal of Interpersonal Violence, in press. Doi: http://dx.doi. org/10.1177/0886260520905545

Castillo Saavedra, E.F., Bernardo Trujillo, J. v. \& Medina Reyes, M. A. (2018). Gender violence and self-esteem in women from the Huanja. Horizonte Médico (Lima), 18(2), 47-52. Doi: https://dx.doi.org/10.24265/ horizmed.2018.v18n2.08

Di Franco, N., Ferreyra, N. \& Di Franco, M. (2016). Prácticas educativas en matemática desde perspectivas sociopolíticas. La ESI y los DDHH como ambientes de aprendizaje y como escenarios de investigación. PRAXIS educativa, 20(2), 41-54. Doi: http://dx.doi.org/10.19137/ praxiseducativa-2016-200205

Difabio de Anglat, H. (2005). El tratamiento de la información en el proceso investigativo. Triangulación. Documento de cátedra. Mendoza: Universidad del Aconcagua.

Escalante Gómez, E. y Páramo, M. A. (octubre 2011). TextSTAT 2.8: Análisis de textos. En: E. Escalante Gómez y M. A. Páramo (Comp.) Aproximación al análisis de datos cualitativos: Aplicación en la práctica investigativa (717- 736). Mendoza, Argentina: Editorial de la Universidad del Aconcagua.

Escoto Sainz, Y.; González Castro, M.; 
Violencia Psicológica en la relación de noviazgo en estudiantes universitarios que inician...

Muñoz Sandoval, A. y Salomón Quintana Y. (2007). Violencia en el Noviazgo Adolescente. Revista Internacional de Psicología, 8(2), 1-33. Recuperado de: http://psicologiarevista.99k. org/violencia $\% 20$ en $\% 20 \mathrm{el} \% 20$ noviazgo $\% 20$ adolescente.pdf

García-Díaz, V., Fernández-Feito, A., Bringas-Molleda, C., RodríguezDíaz, F. \& Lana, A. (2020). Tolerancia de la violencia en la pareja y las actitudes sexistas entre estudiantes universitarios/ as de ciencias de la salud de tres universidades españolas. Gaceta Sanitaria, 34(2), 179-185. Doi: https://dx.doi.org/10.1016/j. gaceta.2019.01.003

Gürtler, L. y Günter, L. H. (2007). Modos de pensar y estrategias de la investigación cualitativa. Liberabit, 13, 37-52. Recuperado de: http:// www.scielo.org.pe/pdf/liber/ v13n13/a05v13n13.pdf

Guzmán-González, M.; García, S.; Sandoval, B.; Vásquez, N.; y Villagrán, C. (2014). Violencia psicológica en el noviazgo en estudiantes universitarios chilenos: diferencias en el apego y la empatía diádica. Interamerican Journal of Psychology, 48(3), 338-346. Recuperado de: http://www. redalyc.org/pdf/284/28437897010. pdf

Hernández Sampieri, R.; Fernández Collado, C. y Baptista Lucio, M. P. (2010). Metodología de la Investigación. México, D.F.: Mc Graw- Hill.

Hernando Gómez, A.; García Rojas, A. y
Montilla Coronado, M. (2012). Exploración de las actitudes y conductas de jóvenes universitarios ante la violencia en las relaciones de pareja. Revista Complutense de Educación, 23(2), 427-44. Recuperado de: http://www.uhu.es/ angel.hernando/documentos/2012 Revista_Complutense_de Educacion.pdf

Hüning, M. (2000/2014). TextSTAT (Versión 2.9c) [Software de Ordenador]. Disponible en http:// www.niederlandistik.fu-berlin.de/ textstat/

Marcos Santiago, M. e Isidro de Pedro, A. I. (2019). El fantasma del control y los celos: violencia de género durante el noviazgo. International Journal of Developmental and Educational Psychology, 2(1), 411-424. Recuperado de: http://www.redalyc. org/articulo.oa? id=349860126038

Moura Lourenço, L.; Nunes Baptista, M.; Aparecida Almeida, A.; Basílio, C.; Mattos Koga, B.; Hashimoto, J.; Kiemy, F.; Stroppa, T.; Monteiro de Castro Bhona, B.F.; Castellani Andrade, G. (2013). Panorama da violência entre parceiros íntimos: Uma revisão crítica da literatura. Interamerican Journal of Psychology, 47(1), 91 99. Recuperado de: http://www. redalyc.org/pdf/284/28426980011. pdf

Olvera Rodríguez, J., Arias López, J y Amador Velázquez, R. (2012). Tipos de violencia en el noviazgo: estudiantes universitarias de la UAEM Zumpango. Revista 
Electrónica de Psicología Iztacala, 15(1), 150-171. Recuperado de: http://www.iztacala.unam. $\underline{\mathrm{mx} / \mathrm{carreras} / \mathrm{psicologia/psiclin} /}$ vol15num1/Vol15No1Art8.pdf

Organización Mundial de la Salud (2003). Informe Mundial sobre la Violencia y la Salud. Cap.1: La violencia como problema mundial de salud pública. Publicación científica $\mathrm{N}^{\circ}$ 120, Washington, DC.

Oviedo Aguilar, M. (2017). Violencia en relaciones de pareja en jóvenes estudiantes universitarios. Revista Varianza, 14, 42-61. Recuperado de: http://www.revistasbolivianas. org.bo/pdf/rv/n14/n14 a06.pdf

Páramo, M. A. y Arrigoni, F. S. (2018). Violencia psicológica en la relación de noviazgo en estudiantes universitarios mendocinos (Argentina). Archivos de Medicina, 18(2), 324-338. Doi: https://doi.org/10.30554/ archmed.18.2.2738.2018

Peña Cárdenas, F.; Zamorano González, B.; Hernández Rodríguez, G.; Hernández González, M. L.; Vargas Martínez, J. y Parra Sierra, V. (2013). Violencia en el noviazgo en una muestra de jóvenes mexicanos. Revista Costarricense de Psicología, 32(1), 27-40. Recuperado de: http://www.redalyc. org/pdf/4767/476748711003.pdf

Pérez-Marco, A., Soares, P., Davó-Blanes, M. \& Vives-Cases, C. (2020). Identifying types of dating violence and protective factors among adolescents in Spain: A qualitative analysis of lights4violence materials. International Journal of Environmental Research and Public Health, 17(7).Doi: http:// dx.doi.org/10.3390/ijerph17072443

Redondo Pacheco, J.; Inglés Saura, C. y García Lizarazo, K. (2017). Papel que juega la edad en la violencia en el noviazgo de estudiantes de la Universidad Pontificia Bolivariana de Bucaramang. Diversitas, 13(1), 41-54. Recuperado de: http:// revistas.usta.edu.co/index.php/ diversitas/article/view/3491/3381

Rey Anacona, C., Martínez Gómez, J. y Londoño Arredondo, N. (2017). Diferencias entre adolescentes del área rural-urbana en malos tratos durante el noviazgo. Diversitas: Perspectivas en Psicología, 13 (2), 159-168. DOI: $10.15332 / \mathrm{s} 1794-$ 9998.2017.0002.02

Rey-Anacona, C.A. (2009) Maltrato de tipo físico, psicológico, emocional, sexual y económico en el noviazgo: Un estudio exploratorio. Acta Colombiana de Psicología, 12(2), 27-36. Recuperado de: http://www. scielo.org.co/ pdf/acp/v12n2a03. pdf

Rey-Anacona, C.A. (2013). Prevalencia y tipos de maltrato en el noviazgo en adolescentes y adultos jóvenes. Terapia psicológica, 31(2), 143154. Recuperado de: http://www. redalyc.org/pdf/785/78526609001. pdf

Rey-Anacona, C.A.; Mateus-Cubides, A.M. y Bayona Arévalo, P.A. (2010) Malos tratos ejercidos por adolescentes durante el noviazgo: diferencias por sexo. 
Violencia Psicológica en la relación de noviazgo en estudiantes universitarios que inician...

Revista Mexicana de Psicología, 27(2), 169-181. Recuperado de: http://www.redalyc.org/articulo. oa? id $=243016324006$

Rodríguez Domínguez, C.; Durán Segura, M. y Martínez-Pecino, R. (2018). Ciberagresores en el noviazgo adolescente y su relación con la violencia psicológica, el sexismo y los celos. Health and Addictions, 18(1), 17-27. Recuperado de: https://idus.us.es/ bitstream/handle/11441/70532/ $\mathrm{C}$ i b e ragres ore s. pdf? sequence $=1 \&$ isAllowed $=y$

Rodríguez Hernández, R.; Riosvelasco Moreno, L. y Castillo Viveros, N. (2018). Violencia en el noviazgo, género y apoyo social en jóvenes universitarios. Escritos de Psicología, 11(1), 1-9. Doi: https:// doi.org/10.5231/psy.writ.2018.2203

Silverman, J.G., Raj, A., Mucci, L.A., \& Hathaway, J.E. (2001). Dating violence against adolescent girls and associated substance use, unhealthy weight control, sexual risk behavior, pregnancy, and suicidality. Journal of the American Medical Association, 266(5), 572579.

Toldos Romero, M.P. (2013). Hombres víctimas y mujeres agresoras: la cara oculta de la violencia entre sexos. España: Cántico. Recuperado de: https://books.google.com.ar/books? $\underline{\mathrm{hl}=\mathrm{es} \& \mathrm{lr}=\& \mathrm{id}=\mathrm{GgQzs} P F e a E c C \& 0}$ $\mathrm{i}=$ fnd\&pg $=$ PA7 $\& \mathrm{dq}=$ hombres + vict imas + mujeres + agresoras + toldos $+r$ omero + \&ots $=1 \mathrm{k} 9 \mathrm{E} 7 \mathrm{ymU} 4 \mathrm{i} \& \mathrm{sig}=\mathrm{f}$ JVAt4HMLQkpRMQKevza2RoB
Qm8\# $\mathrm{v}=$ onepage \&q $=$ hombres $\% 20$

victimas $\% 20 \mathrm{mujeres} \% 20$

agresoras $\% 20$ toldos $\% 20$ romero\& $\mathrm{f}=$ false

Urbiola, I.; Estévez, A.; Iruarrizaga, I.; Momeñe, J.; Jáuregui, P.; Bilbao, M. y Orbegozo, U. (2019). Dependencia emocional en el noviazgo: papel mediador entre la autoestima y la violencia psicológica en jóvenes. Revista española de drogodependencias, 44(1), 13-27. Recuperado de: https://www.aesed. com/upload/files/v44n1 art1.pdf

Valdez Medina, J.L. (1998). La técnica de redes semánticas naturales. En J.L. Valdez Medina, Las redes semánticas naturales, usos y aplicaciones en psicología social. (pp. 65-80). México: Universidad Autónoma del Estado de México.

Valles, M. (2003). El reto de la calidad en la investigación social cualitativa: de la retórica a los planteamientos de fondo y las propuestas técnicas. Reis, 110 (5), 91-114. Recuperado de: http://reis.cis.es/REIS/PDF/ REIS_110_051168261781313.pdf

Vázquez García, V. y Castro, R. (2008). “¿Mi novio sería capaz de matarme?" Violencia en el noviazgo entre adolescentes de la Universidad Autónoma Chapingo, México. Revista Latinoamericana de Ciencias Sociales, Niñezy Juventud, 6 (2), 709-738. Recuperado de: http://www.scielo.org.co/pdf/rlcs/ v5n2/v6n2a08.pdf

Velázquez Rivera, G. (2011). La violencia durante el noviazgo en adolescentes. Elementos, 82(18), 
39-43. Recuperado de: http://www. elementos.buap.mx/ num82/pdf/39. pdf

White, J. \& Koss, M. (1991). Courtship violence: Incidence in a national sample of higher education students. Violence and Victims, 6, 247-256.

Wolfe, D.A.; Crooks, C.V. \& Hughes, R.
(2011) La Cuarta R: Un Programa Escolar de Prevención de la Violencia en las relaciones de Pareja en la Adolescencia. Psychosocial Intervention, 20(2), 193-200. Recuperado de: http://redalyc.org/ articulo.oa? id=179819285007

\section{Notas al final}

${ }^{1}$ Investigación financiada por el Instituto de Investigaciones de la Facultad de Psicología, Universidad del Aconcagua

${ }^{2}$ Valor M total de cada palabra definidora es un puntaje que se obtiene multiplicando la frecuencia de la palabra por la jerarquía obtenida, es decir, el valor asignado por los alumnos a esa palabra. Constituye el peso semántico de cada palabra definidora.

${ }^{3}$ Para la promoción de la igualdad de género, toda referencia a personas o colectivos incluida en este documento hace referencia al género gramatical neutro, incluyendo, por lo tanto, la posibilidad de referirse tanto a mujeres como a hombres. 
Anexo

Líneas temáticas y preguntas disparadoras

1. Aprendizaje a partir de la experiencia de victimización de las/los participantes: ¿Qué les dejó esta experiencia?

2. Cómo afecta la VPen futuras relaciones: ¿estas conductas / actitudes les afectaron emocionalmente cuando la relación de noviazgo ya había terminado? ¿De qué manera?

3. Cómo afecta la VP en la relación actual: ¿estas conductas / actitudes les afecta emocionalmente en la relación de noviazgo actual? ¿De qué manera?

\section{Consideración de la VP como problema:} ¿consideran que la VP en el noviazgo es un problema? ¿Qué tipo de problema?

5. Definición de VP: ¿qué entienden por violencia psicológica (VP)?

6. Diferencias entre VP y violencia física: ¿existen diferencias entre la VPy la violencia física? ¿Cuáles?

7. Diferencias generacionales al considerar la VP: ¿creen que lo de la VP se ha presentado en diferentes generaciones?

8. Emociones que genera la VP: estas conductas / actitudes ¿qué emociones le generaban / generan?
9. Experiencia de victimización en las/ los participantes: en alguna relación de noviazgo, ¿han experimentado conductas o actitudes de VP? ¿Cuáles? ¿De parte de quién? (¿de ambos?, ¿de tu pareja hacia vos?, ¿de vos hacia tu pareja?)

10. Factores que influyen en la VP ¿qué es lo que influye en la VP?

11. Hombre más violento que la mujer: ¿quién creen que presenta más violencia? ¿el hombre o la mujer o ambos?

12. Manifestación de la VP: ¿estas conductas / actitudes afectaban / afectan la relación de noviazgo? ¿De qué manera?

13. Razones para ejercer/sufrir VP: ¿por qué creen que algunas personas ejercen/ sufren VP en su noviazgo?

14. Reacciones frente a la VP: ¿qué hacía la pareja cuando sucedía esto?

15. Recursos de ayuda: ¿a quiénes acudir en estas situaciones de VP?

16. Síntesis/reflexión final: ¿Qué podrían concluir luego de esta charla grupal sobre la VPN?

17. VP más dañina que la violencia física: ¿consideran que la VP es más dañina que la violencia física? ¿Por qué? 